\title{
Upregulation of microRNA-34a enhances the DDP sensitivity of gastric cancer cells by modulating proliferation and apoptosis via targeting MET
}

\author{
ZHANDONG ZHANG $^{1}$, YE KONG ${ }^{1}$, WEI YANG ${ }^{1}$, FEI MA $^{1}$, YONGLEI ZHANG ${ }^{1}$, \\ SHEQING JI ${ }^{1}$, ER-MIN MA ${ }^{2}$, HONGXING LIU ${ }^{1}$, YONGSHUN CHEN ${ }^{3}$ and YAWEI HUA ${ }^{1}$ \\ ${ }^{1}$ Department of General Surgery, Affiliated Tumor Hospital of Zhengzhou University, Henan Cancer Hospital, \\ Zhengzhou, Henan 450008; ${ }^{2}$ Surgical Oncology, The People Hospital of Zhengzhou, Zhengzhou, \\ Henan 450000; ${ }^{3}$ Department of Radiotherapy, Affiliated Tumor Hospital of Zhengzhou \\ University, Henan Cancer Hospital, Zhengzhou, Henan 450008, P.R. China
}

Received February 17, 2016; Accepted July 14, 2016

DOI: $10.3892 /$ or.2016.5016

\begin{abstract}
Cisplatin (DDP) based chemotherapy is still the main strategy of human gastric cancer (GC) treatment. However, drug resistance is a major obstacle for DDP chemotherapy. Recent studies indicated that the resistance could be modulated by the regulation of dysregulated microRNAs (miRs). Previous study also found miR-34a was associated with cell proliferation and apoptosis in human GC; however, the relationship between miR-34a and DDP resistance still remains unexplored. The purpose of this study was to investigate whether miR-34a is associated with DDP resistance in human GC cells. Our study found that the expression of miR-34a was significantly decreased in DDP resistance human GC tissues and DDP resistance human GC SGC7901/DDP cells compared with normal GC tissues and cells. Upregulation of miR-34a enhanced the DDP sensitivity of SGC7901/DDP cells to DDP through the inhibition of cell proliferation and induction of cell apoptosis; on the other hand downregulation of miR-34a could weaken the DDP sensitivity of SGC7901 cells to DDP. Further study found that MET was a direct target of miR-34a and the regulation of MET could affect the DDP sensitivity of SGC7901/DDP cells. Moreover, our study also indicated that upregulation of miR-34a could decrease the expression of MET in SGC7901/DDP cells. Therefore, our findings suggested miR-34a could modulate human gastric cancer cell DDP sensitivity by regulation of cell prolifera-
\end{abstract}

Correspondence to: Dr Yawei Hua, Department of General Surgery, Affiliated Tumor Hospital of Zhengzhou University, Henan Cancer Hospital, 127 Dongming Road, Zhengzhou, Henan 450008, P.R. China

E-mail: yaweih@yeah.net

Key words: miR-34a, human gastric cancer, SGC7901, SCG7901/ DDP, DDP sensitivity, MET tion and apoptosis via targeting MET, potentially benefiting human GC treatment in the future.

\section{Introduction}

Gastric cancer (GC) is one of the most common cancers worldwide, and GC is the 2 nd leading cause of cancer death $(1,2)$. Currently surgery is the main treatment for early GC; however recurrence is very common in GC patients after surgery $(3,4)$. Therefore, the combination of surgery and chemotherapy is a new strategy for the treatment of GC $(5,6)$. Although chemotherapy has developed rapidly in recent years, cisplatin (DDP) still remains the most widely used for GC treatment (7-9). However, the strategy of DDP-based chemotherapy and surgery combination is limited by drug resistance, on the other hand continuous and multiple DDP administration often caused serious side effects (10). Therefore, improving the sensitivity of human GC cell to DDP is a critical point for resolving the challenge. Previous studies have indicated that cell proliferation, cell apoptosis and DNA repair may play important roles in drug resistance $(11,12)$, and the drug resistance always associated with the abnormal activation of some signal pathways (13-16).

It is well known that microRNAs (miRs) are a class of short noncoding RNAs. miRs consist of approximately 19-24 nucleotides, and they were involved in post-transcriptional gene regulation or degradation by direct regulation of target messenger RNA or translational repression $(17,18)$. Previous studies reported that aberrant miRs were found in many types of human tumors (19-23), and some miRs were related to chemoresistance of DDP in human tumor cells (24-29). Therefore, the combination of miRs and chemotherapy may play important roles in the treatment of human cancer. Previous study indicated that miR-34a was significantly decreased in human gastric cancer (30); however, whether the regulation of miR-34a was associated with DDP drug resistance is still unknown.

In this study, we found the expression of miR-34a was significantly downregulated in DDP-resistant human GC 
tissue samples and cell line. Moreover, upregulation of miR-34a could inhibit the cell proliferation of DDP resistant SGC7901/DDP cells by the induction of cell apoptosis through targeting the MET gene. These finds could help to resolve the DDP resistance in the treatment of human GC.

\section{Materials and methods}

Cell culture. SGC7901 cells were purchased from ATCC. SGC7901/DDP cells were purchased from Nanjing KeyGEN Biotech. Cells were cultured in Roswell Park Memorial Institute (RPMI)-1640 medium supplemented with FBS (10\%), penicillin (100 IU/ml) in and streptomycin (100 IU/ml). RPMI-1640, FBS, penicillin and streptomycin were all purchased from Invitrogen. To maintain the DDP resistant phenotype of SGC7901/DDP, SGC7901/DDP cells were cultured with DDP, the concentration of DDP is $1 \mu \mathrm{g} / \mathrm{ml}$. Before our study, SGC7901/DDP cells were cultured without DDP for 1 week.

Clinical samples. A total of 38 histopathologically confirmed GC patient samples were obtained from the Affiliated Tumor Hospital of Zhengzhou University. All these patients received DDP-based chemotherapy before. The GC tissue samples were collected from these advanced patients who underwent 2 cycles of DDP chemotherapy. The study was approved by the institutional review boards of the Affiliated Tumor Hospital of Zhengzhou University, and written informed consent was obtained from each patient.

Cell viability assay. The cell viability was evaluated by methyl thiazolyl tetrazolium (MTT) assay. First $~ 5000$ cells were seeded into 96-well plate for $12 \mathrm{~h}$, and then the cells were treated with serial concentrations of DDP. At $48 \mathrm{~h}$ after DDP treatment, MTT solution was added to each well (1:10), the final concentration of MTT was $0.5 \mathrm{mg} / \mathrm{ml}$, then the cells were incubated for $4 \mathrm{~h}$. The absorbance was read at $570 \mathrm{~nm}$ with a microplate reader (MD). Each experiment was performed in triplicate and the data are presented as mean $\pm \mathrm{SD}$.

Transfections. miR-34a mimic, miR-34a inhibitor and control were all purchased from Invitrogen. Cells were cultured in RPMI-1640 medium with 10\% FBS for $24 \mathrm{~h}$, then miR-34a or miR-34a inhibitor were transfected with Lipofectamine 2000 in antibiotic-free Opti-MEM medium according to the manufacturer's instruction. The final concentration of miR-34a mimic and miR-34a inhibitor was $10 \mathrm{nM}$. At $6 \mathrm{~h}$ after transfection, the medium was changed to RPMI-1640 with $10 \%$ FBS MET siRNA was purchased from Santa Cruz Biotechnology, Inc. (Santa Cruz, CA, USA). The protocol used for MET knockdown has been previously described (30).

RNA extraction and miR-34a examination. SGC-7901 or SGC-7901/DDP cells were treated and cultured, total RNA was extracted from the cells using TRIzol reagent as described by the manufacturer. TaqMan miRNA assays (Applied Biosystems) was performed to examine the expression of miRNA-34a. Briefly, total RNA was reverse transcribed by using a miRNA-specific looped RT primer (Applied Biosystems). Each TaqMan miRNA Reverse Transcription kit was used for a corresponding miRNA. Then qPCR was performed to evaluate the level of miR-34a using TaqMan Universal PCR Master Mix with miRNA-specific TaqMan minor groove binder probes. In the present study RNA U6 was used as an internal control. The relative expression of miR-34a was calculated using the comparative Cycle threshold method. Each experiment was performed in triplicate and the data are presented as mean $\pm \mathrm{SD}$.

Flow cytometric analysis of apoptosis. SGC-7901 or SGC-7901/DDP cells were transfected with inhibitor or mimic and treated with DDP for $48 \mathrm{~h}$. The DDP concentration of SGC7901 was $0.5 \mu \mathrm{g} / \mathrm{ml}$ and for SGC7901/DDP was $5 \mu \mathrm{g} / \mathrm{ml}$. Cells were harvested and submitted to FACS analysis. The cells were double stained with FITC-Annexin V and Propidium iodide (PI), and then the stained cells were analyzed with flow cytometry equipped with CellQuest software. Each experiment was performed in triplicate and the data are presented as mean \pm SD.

Luciferase assay. The 3'-untranslated region (3'UTR) sequences of MET was cloned into the downstream region of the firefly luciferase gene, mutant (Mut) MET was used as control. Human gastric cancer SGC7901 cells were cultured for $24 \mathrm{~h}, \mathrm{pGL} 3$-MET-3'-UTR (WT or Mut) and miR-34a mimic or miRNA mimic control were co-transfected by Lipofectamine 2000. Cells were cultured for another $48 \mathrm{~h}$, and then the cells were harvested for luciferase assay. The Dual-Luciferase Reporter Assay System was used to measure luciferase activity as described by the manufacturer (Promega). The relative luciferase activity was normalized to the renilla luciferase expression. Each experiment was performed in triplicate and the data are presented as mean \pm SD.

Western blot analysis. Human GC tissues and cells were harvested and protein was extracted by using RIPA lysis buffer. Total protein $(40 \mu \mathrm{g})$ was separated on 15\% SDS-PAGE gels and electrophoretically transferred onto a PVDF membrane (GE). The PVDF membrane was blocked with 5\% non-fat dry milk for $\sim 2 \mathrm{~h}$, and then the PVDF membrane was incubated with specific primer antibodies MET, and GAPDH for $2 \mathrm{~h}$. The PVDF membrane was washed with TBST 3 times, and incubated with horseradish peroxidase-linked second antibody for another $1 \mathrm{~h}$. The PVDF membranes were washed and the proteins were visualized using ECL chemiluminescence and exposed to X-ray film. Each experiment was performed in triplicate.

Real-time PCR to detect the mRNA of MET. Total RNA was isolated from treated gastric cancer tissues and cells using TRIzol reagent as described by the manufacturer (Invitrogen). cDNA was synthesized from total RNA using a Reverse Transcription kit (Applied Biosystems). The primer and probe sequences were: GAPDH primer (F: 5'-TCGACAGTCAGC CGCATCTTCTTT-3'; R: 5'-ACCAAATCCGTTGACTCCG ACCTT-3'; and probe: 5'-6FAM-AGCCACATCGCTCA GACACCATGGG-TAMRA-3'); MET primer (F: 5'-TGC AGCGCGTTGACTTATTCATGG-3'; R: 5'-GAAACCACA ACCTGCATGAAGCGA-3'; and probe: 5'-6FAM-AGGAGA CCTCACCATAGCTAATCTTGGG-TAMRA-3'); Real-time 


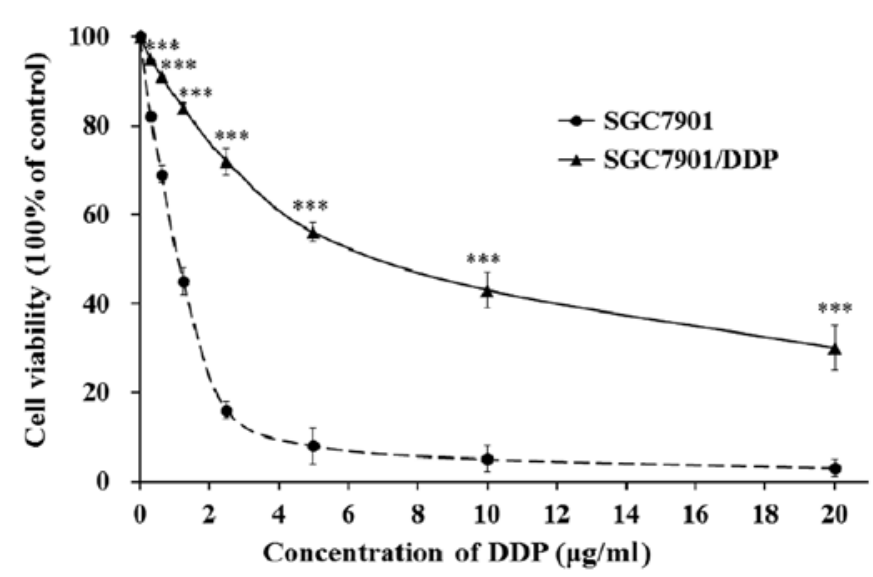

Figure 1. Survival curves of human gastric cells cultured with different concentrations of DDP. Cell viability was measured by MTT assay. The data are expressed as the mean \pm SD from at least 3 separate experiments. ${ }^{* * *} \mathrm{p}<0.001$.

PCR was performed as follows, $95^{\circ} \mathrm{C}$ for $10 \mathrm{~min}$ followed by 40 cycles at $95^{\circ} \mathrm{C}$ for $15 \mathrm{sec}$ and at $56^{\circ} \mathrm{C}$ for $60 \mathrm{sec}$. GAPDH was used as an internal control. Each experiment was performed in triplicate and the data are presented as mean $\pm \mathrm{SD}$.

Statistical analysis. The data are expressed as the mean \pm SD from at least 3 separate experiments. Statistical analysis was performed by two-tailed Student's t-tests using SPSS 13.0 (SPSS Inc., Chicago, IL, USA) to evaluate the significance of differences between groups. $\mathrm{p}<0.05$ was defined as statistically significant.

\section{Results}

DDP resistance of SGC7901/DDP cells. In order to check the DDP resistance of SGC7901/DDP cells, we evaluated the cell viability of parental SGC7901 cells and DDP resistant SGC7901/DDP cells when exposed to different concentrations of DDP. SGC7901 and SGC7901/DDP cells were cultured and treated with different concentrations of DDP for $48 \mathrm{~h}$; the cell viability was evaluated by MTT assay. As shown in Fig. 1, when exposed to DDP the cell viability of both SGC7901 cells and SGC7901/DDP cells decreased dose-dependently. The results also indicated that SGC7910/DDP cell lines showed an acquired resistance to DDP compared with the parental SGC7901 cell lines.

miR-34a was downregulated in GC patients with DDP resistance and DDP-resistant SGC7901/ DDP cells. Previous study indicated that the expression of miR-34a was downregulated in human gastric cancer tissues and SGC7901 cell lines (30). To further investigate whether miR-34a was associated with human gastric cancer DDP resistance, we measured the expression of miR-34a in 38 GC tissues from advanced patients who underwent 2 cycles of DDP chemotherapy. Response to chemotherapy was evaluated as previous described (31); defined as complete remission (CR), partial remission (PR), stable disease (SD), and progressive disease (PD). In these patients, 11 patients acquired PR, 12 patient acquired SD, 15 patients acquired PD and no patients acquired CR. The
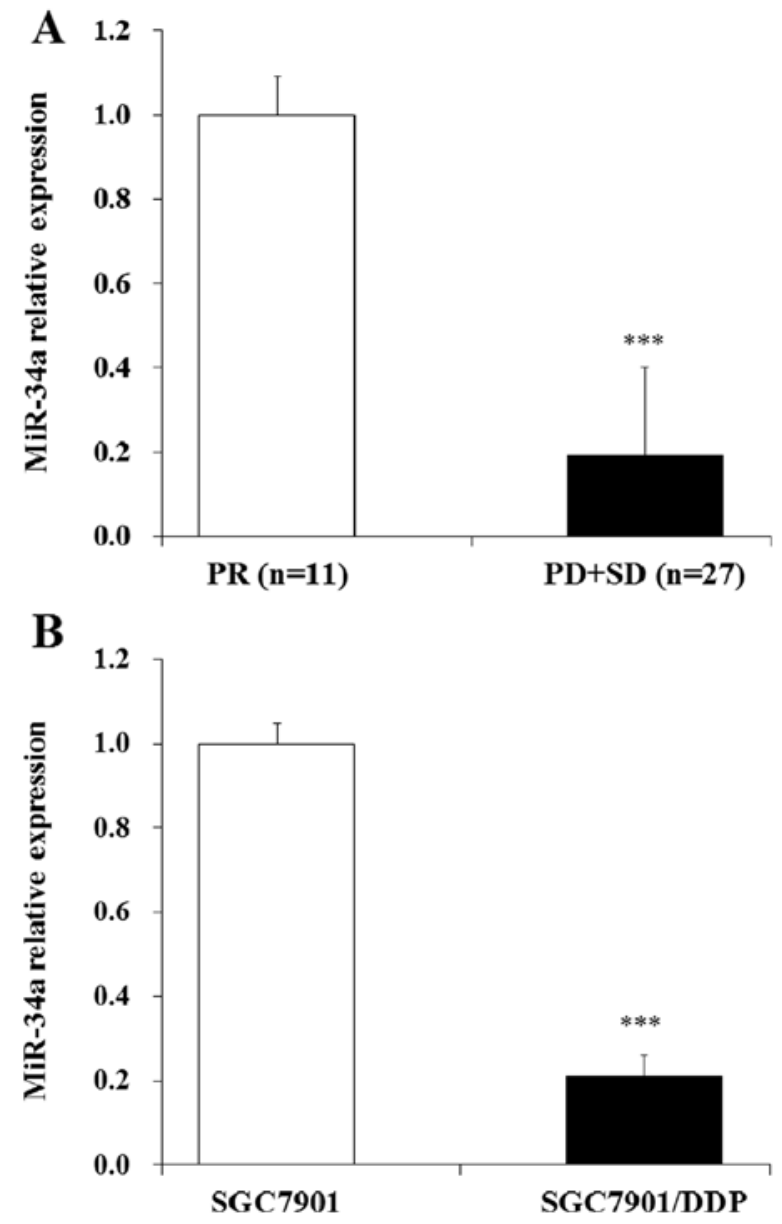

Figure 2. The expression level of miR-34a in human GC tissues and GC cells. The miR-34a level was measured by qRT-PCR assay. The data are expressed as the mean \pm SD from at least 3 separate experiments. ${ }^{* * *} \mathrm{p}<0.001$.

expression of miR-34a in these tissue samples was evaluated by qRT-PCR assay, in our study we found that the expression of miR-34a was downregulated in SD, PD group compared with the PR group (Fig. 2A). Then we tested the level of miR-34a in DDP resistant GC SGC7901/DDP cells, the results indicated that the expression of miR-34a was also downregulated in SGC7901/DDP cells compared with SGC7901 cells (Fig. 2B). These results showed that miR-34a was downregulated both in clinical GC tissues and DDP-resistant GC cells. We hypothesized that the regulation of miR-34a may play important roles in DDP resistant human GC cells.

Manipulation of miR-34a expression in human gastric cancer cells. In order to investigate the biological roles of miR-34a in human gastric cancer cells, we selectively regulated the expression of miR-34a by miR-34a mimic or inhibitor transfection. The qRT-PCR results indicated that the level of miR-34a in SGC7901/DDP cells was significantly increased after the transfection of miR-34a mimic compared with miR-control or parental SGC7901/DDP cells (Fig. 3A). The results also indicated that the level of miR-34a in SGC7901 cells was significantly decreased with transfection of the cells with miR-34a inhibitor compared with the control and parental cells (Fig. 3B). Therefore, we selectively manipulated the expression of miR-34a by mimic or inhibitor transfection in this study. 

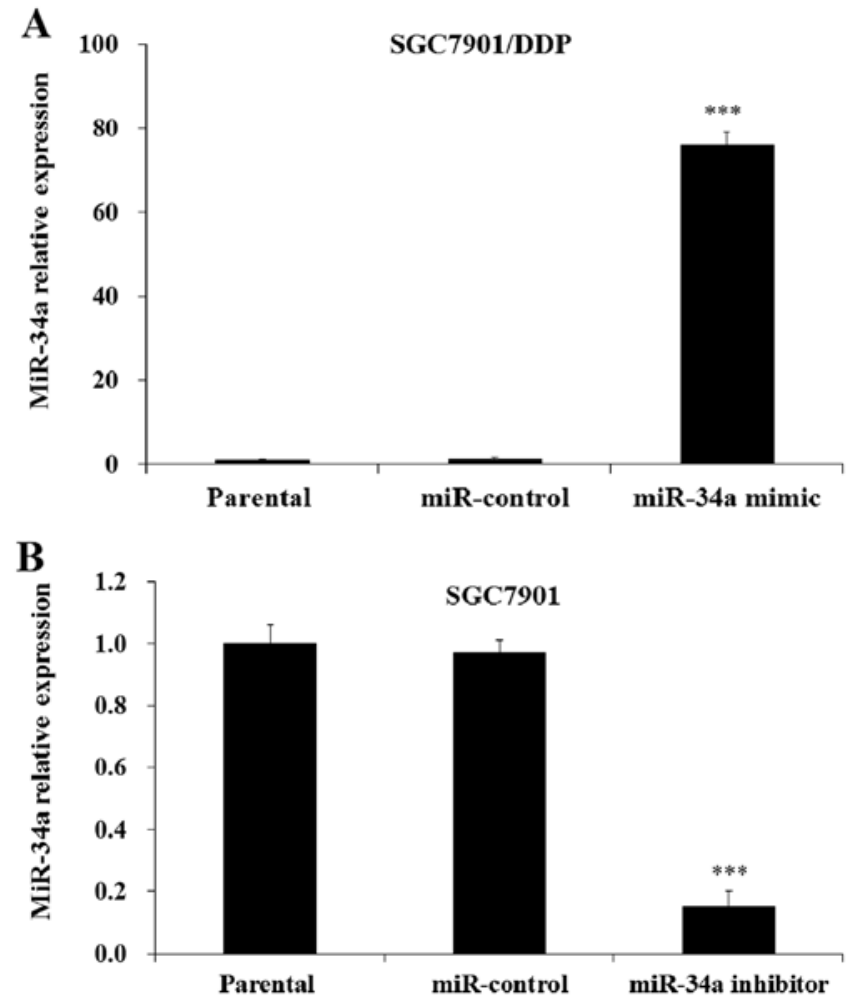

Figure 3. The expression level of miR-34a in mimic or inhibitor transfected human GC cells. (A) The level of miR-34a in SGC7901/DDP cells. (B) The level of miR-34a in SGC7901 cells. The data are expressed as the mean \pm SD from at least 3 separate experiments. ${ }^{* * *} \mathrm{p}<0.001$.

miR-34a modulates DDP resistance of human gastric cancer cells. To study the biological activity of miR-34a, miR-34a mimic or inhibitor transfected SGC7901/DDP or SGC7901 cells were cultured with DDP; the cell viability was evaluated by MTT assay. The results indicated that transfection of miR-34a mimic significantly decreased the cell viability of SGC7901/DDP cells, while the transfection of mimic control showed no influence compared with parental SGC7901/DDP cells (Fig. 4A). The results also indicated that transfection of miR-34a inhibitor significantly increased the cell viability of SGC7901 cells compared with the control and the parental SGC7901 cells (Fig. 4B). These results demonstrated that miR-34a could modulate the DDP resistance of human gastric cancer cells.

miR-34a modulated the DDP-induced apoptosis in human gastric cancer cells. To investigate whether the regulation of cell viability was through the regulation of cell apoptosis, miR-34a mimic or inhibitor transfected SGC7901/DDP or SGC7901 cells were cultured with DDP, and then the cells were harvested. FACS assay was performed to evaluate cell apoptosis. As shown in Fig. 5 the transfection of miR-34a mimic significantly increased cell apoptosis of SGC7901/DDP cells (Fig. 5A), while the transfection of miR-34a inhibitor decreased apoptosis of SGC7901 cells (Fig. 5B). These results demonstrated that miR-34a modulated the DDP induced human gastric cancer cell apoptosis.

MET is the target gene of miR-34a. Previous study predicted that MET gene was the target gene of miR-34a (30). In our

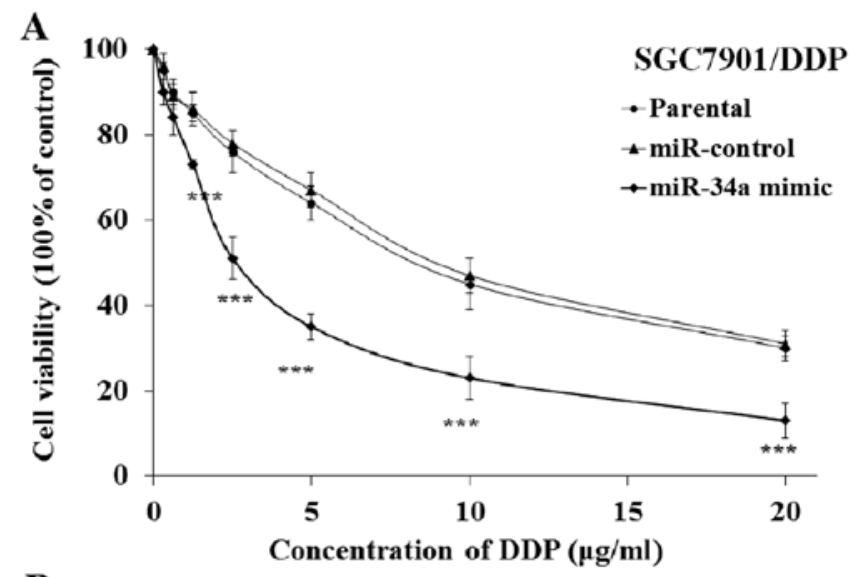

B

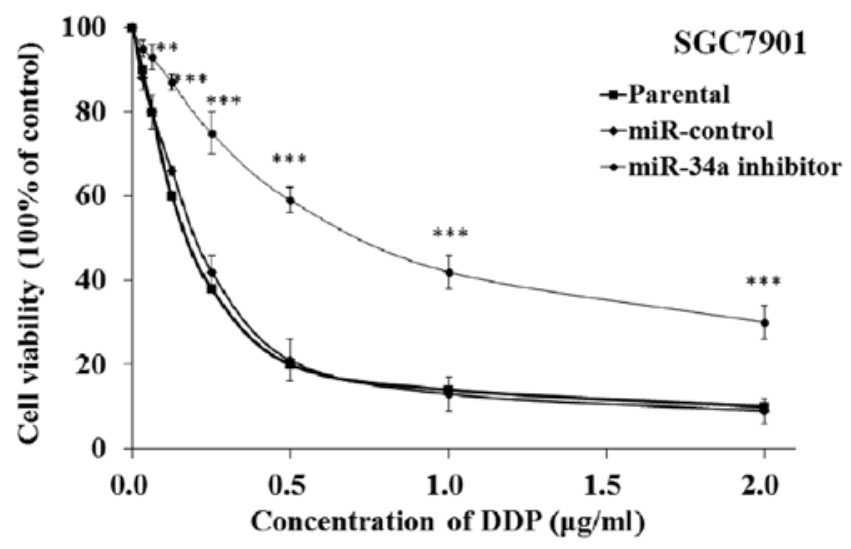

Figure 4. miR-34a regulates the DDP sensitivity of human GC cells. (A) Transfection of miR-34a mimic enhanced the DDP sensitivity of SGC7901/DDP cells. (B) Transfection of miR-34a inhibitor weakened the DDP sensitivity of SGC7901 cells. The data are expressed as the mean \pm SD from at least 3 separate experiments. ${ }^{* * *} \mathrm{p}<0.001$.

study we searched the potential target genes of miR-34a by using TargetScan, PicTar and miRanda program. The predicted results also indicated that MET was a direct target gene of miR-34a (Fig. 6A). To confirm MET was the direct gene of miR-34a, dual luciferase assay was performed. Human gastric cancer SGC7901 cells were co-transfected with pGL3-MET and mimic miR-34a and cultured, then luciferase was tested. The results indicated that the induction of miR-34a led to a reduction of luciferase in SGC7901 cells co-transfected with MET WT, while there was no impact of luciferase in SGC7901 cells co-transfect with MET Mut (Fig. 6B). The result indicated MET is a direct target of miR-34a.

MET was differently expressed in GC patients with DDP resistance and DDP-resistant SGC7901/ DDP cells. Then we examined the expression of MET in human GC patients and GC patients with DDP-resistance. Both the western and RT-PCR results demonstrated that the MET was overexpressed in DDP resistance patients (PD+SD) compared with human GC patients (PR) (Fig. 7A). Further study indicated the level of MET was also upregulated in SGC7901/DDP cells compared with SGC7901 cells (Fig. 7B). These results demonstrated that MET was overexpressed in DDP resistant GC patients and DDP resistant SGC7901/DDP cells. 

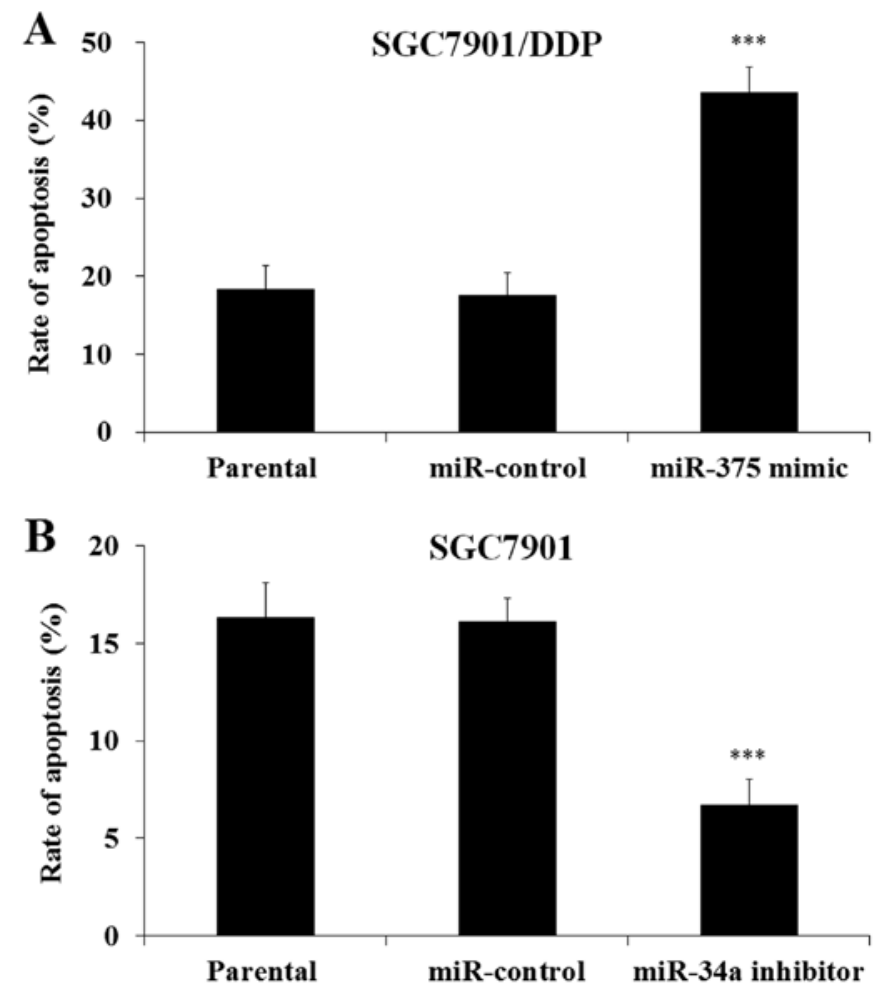

Figure 5. miR-34a regulates cell apoptosis of human GC cells. (A) Transfection of miR-34a mimic induced apoptosis of SGC7901/DDP cells. (B) Transfection of miR-34a inhibitor decreased apoptosis of SGC7901 cells. The data are expressed as the mean \pm SD from at least 3 separate experiments. ${ }^{* * *} \mathrm{p}<0.001$.

\section{A}

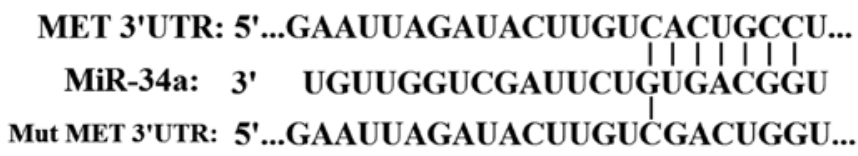

B

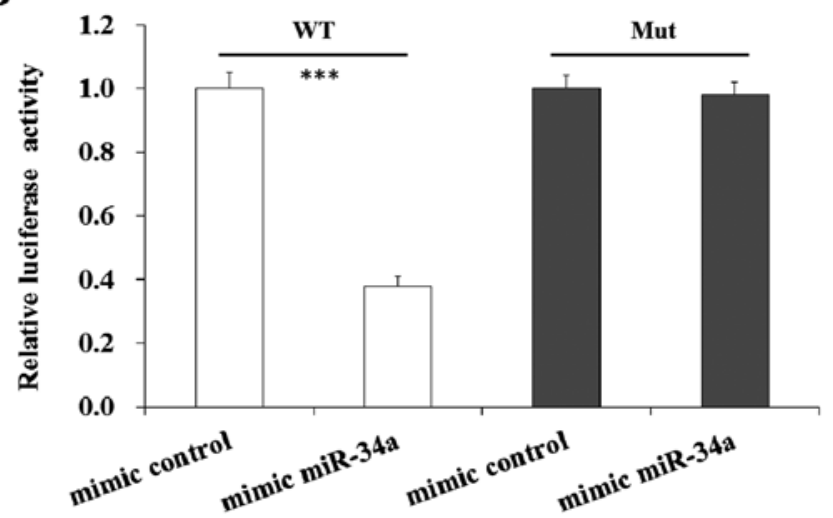

Figure 6. MET is a direct target of miR-34a. (A) Prediction of miR-34a binding sites in the 3'UTRs of human MET gene. (B) MET was a direct target of miR-34a as confirmed by Dual-Luciferase assay. The data are expressed as the mean \pm SD from at least 3 separate experiments. ${ }^{* * *} \mathrm{p}<0.001$.

$M E T$ is related to $D D P$ resistance in $G C$ cells. Then we investigated the relationship between the expression of MET and the DDP resistance in human GC cells. SGC7901/DDP cells transfected with MET siRNA were co-cultured with DDP, the cell viability was evaluated by MTT assay. The
A
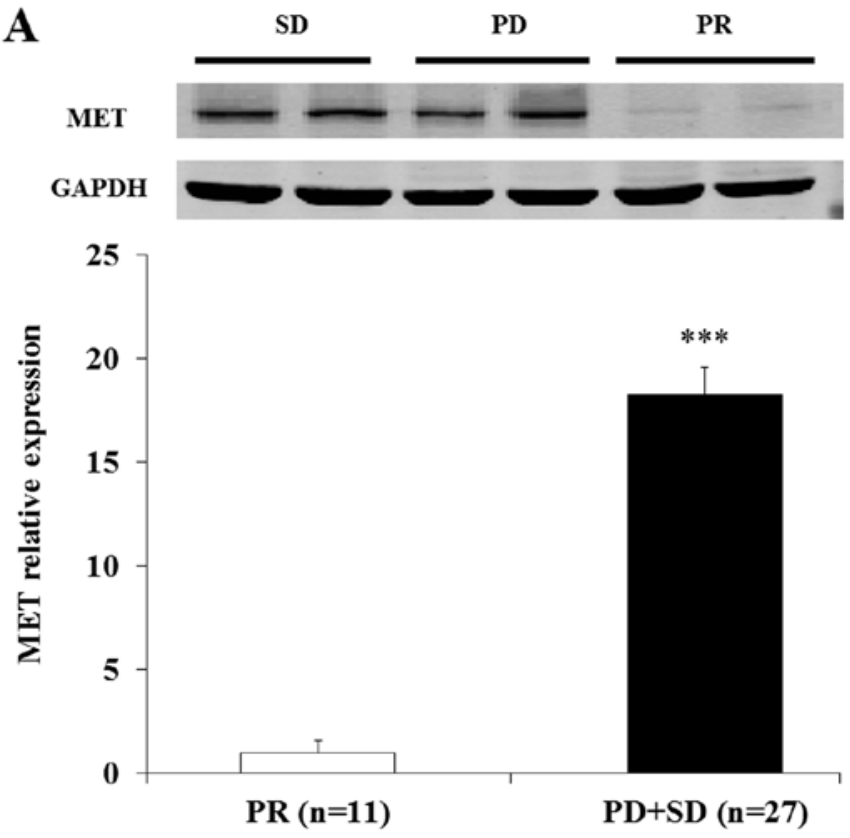

B

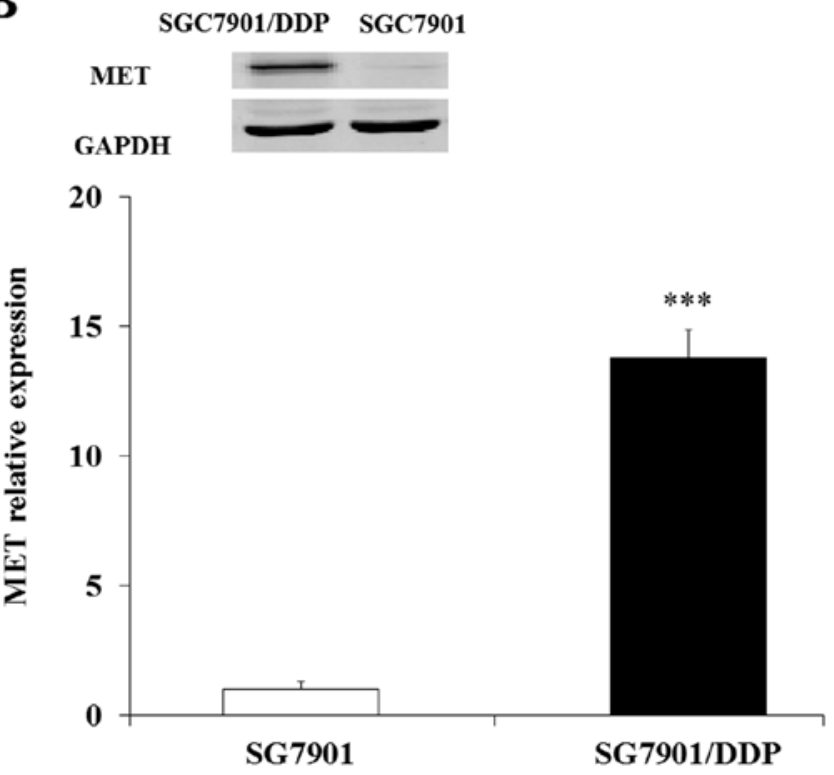

Figure 7. The expression of MET in human GC tissues and GC cells. (A) The expression of MET in human GC tissues was assessed by western blot and RT-PCR. (B) The expression of MET in human GC cells was assessed by western blot and RT-PCR. The data are expressed as the mean \pm SD from at least 3 separate experiments. ${ }^{* * *} \mathrm{p}<0.001$.

results indicated that the cell viability in MET siRNA group was significantly decreased compared with the control and parental group (Fig. 8). Therefore, the expression of MET was associated with the DDP resistance in human GC SGC7901/ DDP cells.

miR-34a modulates DDP resistance by repressing MET. Finally we investigated the DDP resistance mechanism of miR-34a in human GC cells. Our study demonstrated that transfection of miR-34a mimic significantly decreased the level of MET in SGC7901/DDP cells (Fig. 9A) while transfection of miR-34a inhibitor significantly increased the level of MET in SGC7901 cells compared with mimic control and 


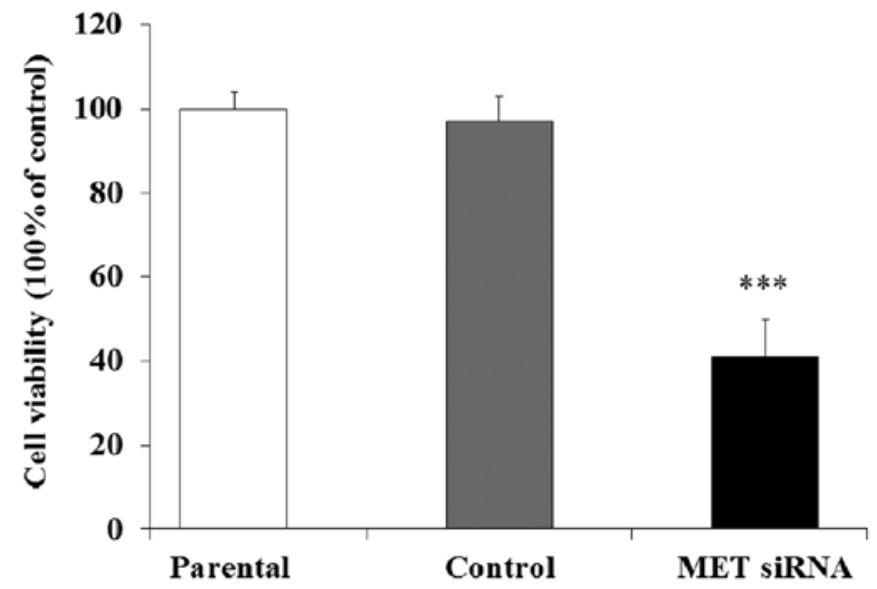

Figure 8. Knockout of MET significantly decreased the cell viability of SCG7901/DDP cells. SGC7901/DDP cells were transfected with MET siRNA and treated with DDP, the cell viability was measured by MTT assay. The data are expressed as the mean \pm SD from at least 3 separate experiments. ${ }^{* * *}$ p $<0.001$.

parental cells (Fig. 9B). Therefore, upregulation of miR-34a led to a decreased expression of MET in human gastric cancer cells.

\section{Discussion}

miR-34 is a class of conserved miRs widespread in mammals (32). miR-34 family consists of miR-34a, miR-34b and miR-34c; the expression of miR-34a is much higher than miR-34b and miR-34c in human organs (33). Previous studies reported that the level of miR-34a was downregulated in many human cancers $(34,35)$. Wei et al reported that miR-34a was also downregulated in human gastric cancer (30), it is reported that miR-34a inhibited proliferation invasion and progression in colon cancer and glioblastoma $(36,37)$. These findings demonstrated that miR-34a plays important roles in human cancers; however the relationship between miR-34a and the DDP sensitivity in human gastric cancer is still unknown.

In order to investigate the relationship between miR-34a and DDP resistance in human gastric cancer, we examined the expression of miR-34a in the DDP resistant GC patient tissue and DDP resistant cell line SGC7901/DDP. The results indicated that the expression of miR-34a was significantly decreased in DDP resistance GC patients and DDP resistant SGC7901/DDP. Further study indicated that upregulation of miR-34a could inhibit the proliferation of SGC7901/DDP cells and induce SGC7901/DDP cell apoptosis when treated with DDP. On the other hand, downregulation of miR-34a increased the cell proliferation of SGC7901 cells and decreased SGC7901 cell apoptosis when treated with DDP. Therefore, the downregulation of miR-34a contributed to the decreased sensitivity of SGC-7901 cells to DDP, and upregulation of miR-34 could increase the sensitivity of SGC-7901/DDP cells to DDP.

By using miR target tools we confirmed MET was a target gene of miR-34a. The dual luciferase assay study indicated that MET is a direct target gene of miR-34a. We also found that the expression level of MET was significantly increased
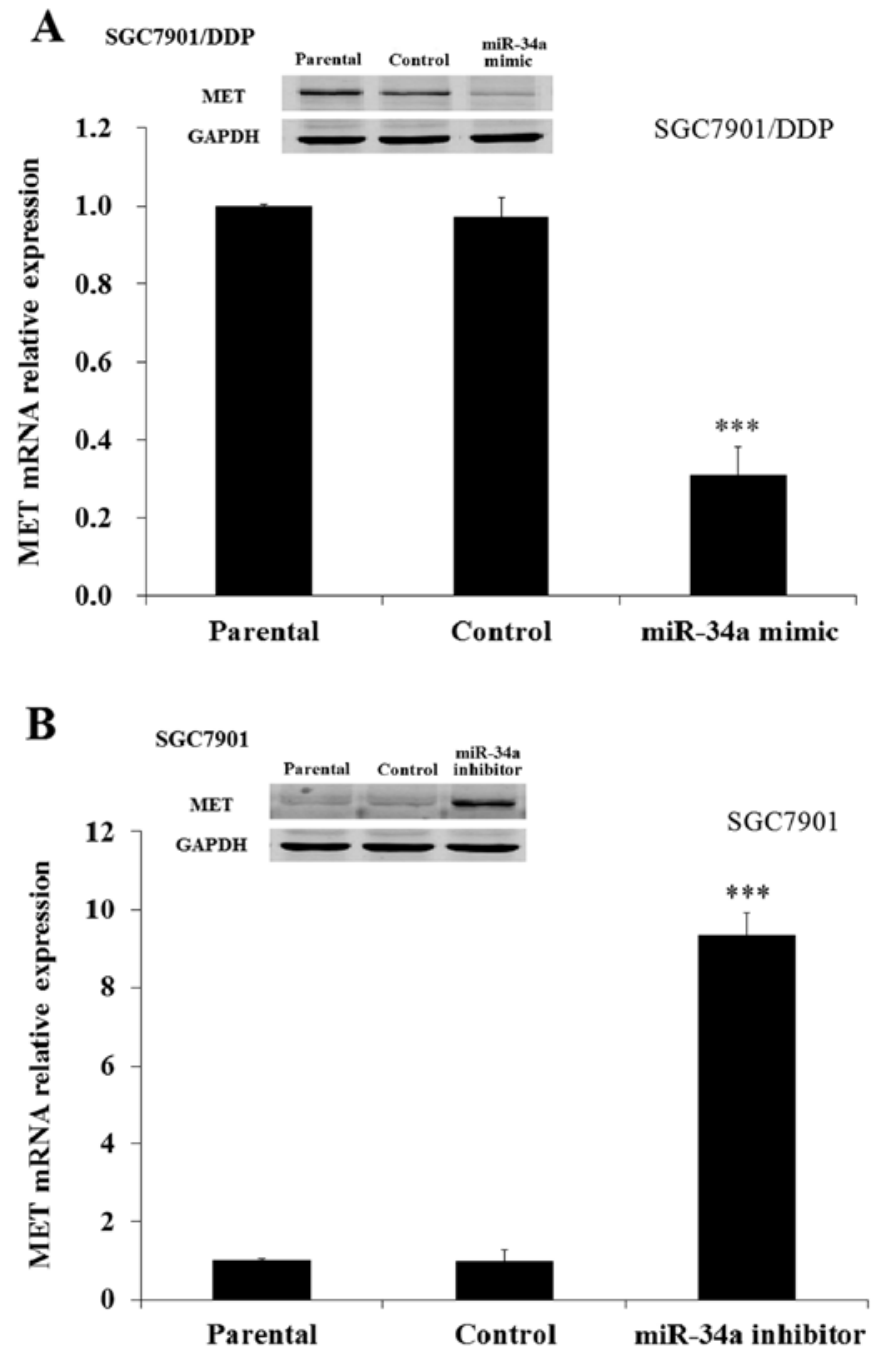

Figure 9. The expression of MET in miR-34a mimic or inhibitor transfected human GC cells. (A) SGC7901/DDP cells were transfected with miR-34a mimic, the expression of MET was assessed by western blot and RT-PCR. (B) SGC7901 cells were transfected with miR-34a inhibitor, the expression of MET was assessed by western blot and RT-PCR. The data are expressed as the mean $\pm \mathrm{SD}$ from at least 3 separate experiments. ${ }^{* * *} \mathrm{p}<0.001$.

in DDP resistant GC patient tissues and cell lines. MET is an oncogene, it was associated with tumor progress and metastasis (38). A previous study indicated that the level of MET was a predictive marker for human colorectal cancer (39). Downregulation of MET lead to the regulation of PI3K signal pathway in human ovarian cancer (40). Dang et al reported that the regulation of miR-34a contributed to hepatocellular cancer malignancy through targeting MET (41). However, there are few studies reporting on the relationship between MET and DDP resistance. We investigated whether the regulation of MET was associated with the DDP resistance by using siRNA knockdown assay, and the results indicated that knockout MET could inhibit the SGC7901/DDP cell proliferation when treated with DDP. These findings suggest upregulation of miR-34 could be sensitive to the DDP resistance of human gastric cancer SGC7901/DDP cells through targeting MET. As a potential concern miR-34a could also non-specifically bind to other mRNAs, which would cause unwanted side effects. To resolve the potential unwanted side effects more studies of miR-34a function are needed. 
In conclusion, the miR-34a expression was significantly downregulated in DDP resistant GC patients and GC cell lines. The upregulation of miR-34a enhanced the sensitivity of human GC cells to DDP treatment through regulation of cell proliferation and apoptosis via the regulation of the MET gene. These results suggested that the combination of miR-34a transfection with DDP treatment might be a new strategy for the treatment of human GC.

\section{References}

1. Ferlay J, Soerjomataram I, Dikshit R, Eser S, Mathers C, Rebelo M, Parkin DM, Forman D and Bray F: Cancer incidence and mortality worldwide: Sources, methods and major patterns in GLOBOCAN 2012. Int J Cancer 136: E359-E386, 2015.

2. Roder DM: The epidemiology of gastric cancer. Gastric Cancer 5 (Suppl 1): 5-11, 2002.

3. Gallo A and Cha C: Updates on esophageal and gastric cancers World J Gastroenterol 12: 3237-3242, 2006.

4. Gunderson LL: Gastric cancer - patterns of relapse after surgical resection. Semin Radiat Oncol 12: 150-161, 2002.

5. Cunningham D, Allum WH, Stenning SP, Thompson JN Van de Velde CJ, Nicolson M, Scarffe JH, Lofts FJ, Falk SJ, Iveson TJ, et al; MAGIC Trial Participants: Perioperative chemotherapy versus surgery alone for resectable gastroesophageal cancer. N Engl J Med 355: 11-20, 2006.

6. Bang YJ, Kim YW, Yang HK, Chung HC, Park YK, Lee KH, Lee KW, Kim YH, Noh SI, Cho JY, et al; CLASSIC trial investigators: Adjuvant capecitabine and oxaliplatin for gastric cancer after D2 gastrectomy (CLASSIC): A phase 3 open-label, randomised controlled trial. Lancet 379: 315-321, 2012.

7. Mesner PW Jr, Budihardjo II and Kaufmann SH: Chemotherapyinduced apoptosis. Adv Pharmacol 41: 461-499, 1997.

8. Kaufmann SH and Earnshaw WC: Induction of apoptosis by cancer chemotherapy. Exp Cell Res 256: 42-49, 2000.

9. Hannun YA: Apoptosis and the dilemma of cancer chemotherapy. Blood 89: 1845-1853, 1997.

10. Kostova I: Platinum complexes as anticancer agents. Recent Patents Anticancer Drug Discov 1: 1-22, 2006

11. Holohan C, Van Schaeybroeck S, Longley DB and Johnston PG: Cancer drug resistance: An evolving paradigm. Nat Rev Cancer 13: 714-726, 2013.

12. Longley DB and Johnston PG: Molecular mechanisms of drug resistance. J Pathol 205: 275-292, 2005.

13. Nakagawa Y, Sedukhina AS, Okamoto N, Nagasawa S, Suzuki N, Ohta T, Hattori H, Roche-Molina M, Narváez AJ, Jeyasekharan $\mathrm{AD}$, et al: NF- $\mathrm{BB}$ signaling mediates acquired resistance after PARP inhibition. Oncotarget 6: 3825-3839, 2015

14. Halilovic E, She QB, Ye Q, Pagliarini R, Sellers WR, Solit DB and Rosen N: PIK3CA mutation uncouples tumor growth and cyclin D1 regulation from MEK/ERK and mutant KRAS signaling. Cancer Res 70: 6804-6814, 2010.

15. Michaelis M, Rothweiler F, Barth S, Cinatl J, van Rikxoort M, Löschmann N, Voges Y, Breitling R, von Deimling A, Rödel F, et al: Adaptation of cancer cells from different entities to the MDM2 inhibitor nutlin-3 results in the emergence of p53-mutated multi-drug-resistant cancer cells. Cell Death Dis 2: e243, 2011.

16. Lin X, Zhang X, Wang Q, Li J, Zhang P, Zhao M and Li X: Perifosine downregulates MDR 1 gene expression and reverses multidrug-resistant phenotype by inhibiting PI3K/Akt/NF- $\kappa \mathrm{B}$ signaling pathway in a human breast cancer cell line. Neoplasma 59: $248-256,2012$

17. Ambros V: The functions of animal microRNAs. Nature 431: 350-355, 2004.

18. Bartel DP: MicroRNAs: Genomics, biogenesis, mechanism, and function. Cell 116: 281-297, 2004.

19. Ma L, Teruya-Feldstein J and Weinberg RA: Tumour invasion and metastasis initiated by microRNA-10b in breast cancer Nature 449: 682-688, 2007.

20. Ma L, Reinhardt F, Pan E, Soutschek J, Bhat B, Marcusson EG, Teruya-Feldstein J, Bell GW and Weinberg RA: Therapeutic silencing of miR-10b inhibits metastasis in a mouse mammary tumor model. Nat Biotechnol 28: 341-347, 2010.

21. Cho WC: MicroRNAs: Potential biomarkers for cancer diagnosis, prognosis and targets for therapy. Int J Biochem Cell Biol 42: $1273-1281,2010$
22. Cho WC: MicroRNAs in cancer - from research to therapy Biochim Biophys Acta 1805: 209-217, 2010.

23. Gabriely G, Teplyuk NM and Krichevsky AM: Context effect: microRNA-10b in cancer cell proliferation, spread and death. Autophagy 7: 1384-1386, 2011.

24. Xiang Y, Ma N, Wang D, Zhang Y, Zhou J, Wu G, Zhao R, Huang H, Wang X, Qiao Y, et al: MiR-152 and miR-185 co-contribute to ovarian cancer cells cisplatin sensitivity by targeting DNMT1 directly: A novel epigenetic therapy independent of decitabine. Oncogene 33: 378-386, 2014.

25. Shang Y, Zhang Z, Liu Z, Feng B, Ren G, Li K, Zhou L, Sun Y, Li M,Zhou J, et al: miR-508-5p regulates multidrug resistance of gastric cancer by targeting ABCB1 and ZNRD1. Oncogene 33: 3267-3276, 2014.

26. Sui C, Meng F, Li Y and Jiang Y: miR-148b reverses cisplatinresistance in non-small cell cancer cells via negatively regulating DNA (cytosine-5)-methyltransferase 1(DNMT1) expression. J Transl Med 13: 132, 2015.

27. Fang L, Li H, Wang L, Hu J, Jin T, Wang J and Yang BB: MicroRNA-17-5p promotes chemotherapeutic drug resistance and tumour metastasis of colorectal cancer by repressing PTEN expression. Oncotarget 5: 2974-2987, 2014.

28. Yu ZW, Zhong LP, Ji T, Zhang P, Chen WT and Zhang CP. MicroRNAs contribute to the chemoresistance of cisplatin in tongue squamous cell carcinoma lines. Oral Oncol 46: 317-322, 2010.

29. Sorrentino A, Liu CG, Addario A, Peschle C, Scambia G and Ferlini C: Role of microRNAs in drug-resistant ovarian cancer cells. Gynecol Oncol 111: 478-486, 2008

30. Wei B, Huang QY, Huang SR, Mai W and Zhong XG: MicroRNA-34a attenuates the proliferation, invasion and metastasis of gastric cancer cells via downregulation of MET. Mol Med Rep 12: 5255-5261, 2015.

31. Du Y, Zhu M, Zhou X, Huang Z, Zhu J, Xu J, Cheng G, Shu Y, Liu P, Zhu W, et al: miR-20a enhances cisplatin resistance of human gastric cancer cell line by targeting NFKBIB. Tumour Biol 37: 1261-1269, 2016.

32. Yao Y, Suo AL, Li ZF, Liu LY, Tian T, Ni L, Zhang WG, Nan KJ, Song TS and Huang C: MicroRNA profiling of human gastric cancer. Mol Med Rep 2: 963-970, 2009.

33. Wong MY, Yu Y, Walsh WR and Yang JL: microRNA-34 family and treatment of cancers with mutant or wild-type p53 (Review). Int J Oncol 38: 1189-1195, 2011.

34. Kumar B, Yadav A, Lang J, Teknos TN and Kumar P: Dysregulation of microRNA-34a expression in head and neck squamous cell carcinoma promotes tumor growth and tumor angiogenesis. PLoS One 7: e37601, 2012.

35. Shen Z, Zhan G, Ye D, Ren Y, Cheng L, Wu Z and Guo J: MicroRNA-34a affects the occurrence of laryngeal squamous cell carcinoma by targeting the antiapoptotic gene survivin. Med Oncol 29: 2473-2480, 2012.

36. Li Y, Guessous F, Zhang Y, Dipierro C, Kefas B, Johnson E, Marcinkiewicz L, Jiang J, Yang Y, Schmittgen TD, et al: MicroRNA-34a inhibits glioblastoma growth by targeting multiple oncogenes. Cancer Res 69: 7569-7576, 2009.

37. Cho WC: OncomiRs: The discovery and progress of microRNAs in cancers. Mol Cancer 6: 60, 2007.

38. Lutterbach B, Zeng Q, Davis LJ, Hatch H, Hang G, Kohl NE, Gibbs JB and Pan BS: Lung cancer cell lines harboring MET gene amplification are dependent on Met for growth and survival. Cancer Res 67: 2081-2088, 2007.

39. Takeuchi H, Bilchik A, Saha S, Turner R, Wiese D, Tanaka M, Kuo C, Wang HJ and Hoon DS: c-MET expression level in primary colon cancer: A predictor of tumor invasion and lymph node metastases. Clin Cancer Res 9: 1480-1488, 2003.

40. Sawada K, Radjabi AR, Shinomiya N, Kistner E, Kenny H, Becker AR, Turkyilmaz MA, Salgia R, Yamada SD, Vande Woude GF, et al: c-Met overexpression is a prognostic factor in ovarian cancer and an effective target for inhibition of peritoneal dissemination and invasion. Cancer Res 67: 1670-1679, 2007.

41. Dang Y, Luo D, Rong M and Chen G: Underexpression of miR-34a in hepatocellular carcinoma and its contribution towards enhancement of proliferating inhibitory effects of agents targeting c-MET. PLoS One 8: e61054, 2013. 